\title{
Correction to: Fluid Mechanics Applied to Medicine
}

\section{Correction to:}

\section{A. Pozo Álvarez, Fluid Mechanics Applied to Medicine, SpringerBriefs in Computational Mechanics, https://doi.org/10.1007/978-3-030-60389-2}

The initially published version of author's last name in Refs. 3, 4, 9 of Chap. 3, Refs. 22, 23, 30, 33 of Chap. 4 and Refs. 2, 3, 6, 8, 13, 15 of Chap. 5 were incorrect. The author's last names are corrected in References. The erratum chapters and the book have been updated with the change.

The updated versions of these chapters can be found at https://doi.org/10.1007/978-3-030-60389-2_3 https://doi.org/10.1007/978-3-030-60389-2_4 https://doi.org/10.1007/978-3-030-60389-2_5 